\title{
Políticas Públicas de Saúde voltadas ao Câncer de Colo de Útero no Brasil: revisão de literatura
}

\author{
Public Health Policies for Cervical Cancer in Brazil: literature review \\ Políticas de Salud Pública para el Cáncer Cervicouterino en Brasil: revisión de la literatura \\ Lucrecia Aline Cabral Formigosa ${ }^{1 *}$, Marcos Valério Santos da Silva ${ }^{1}$.
}

\begin{abstract}
RESUMO
Objetivo: Investigar as políticas públicas de saúde voltadas para a promoção e prevenção do câncer de colo de útero (CCU), disponíveis na literatura no período de 2000 a 2020. Métodos: Trata-se de um estudo de revisão integrativa das bases de dados National Library of Medicine (PUBMED), Scientific Electronic Library Online (SciELO) e Biblioteca Virtual em Saúde (BVS), publicados a partir de 2000, nos idiomas inglês, português e espanhol. Excluíram-se os trabalhos repetidos e aqueles que não respondiam à pergunta de pesquisa proposta. A coleta de dados ocorreu durante o mês de janeiro/2021. Resultados: Foi possível abordar a temática do câncer cervical, no que concerne à gestão do cuidado com ênfase na atenção primária à saúde, apresentando a evolução das políticas públicas implantadas no decorrer de 20 anos. Considerações finais: As publicações revisadas demonstraram, que ao longo do período estudado, houve um esforço governamental em implementar medidas preventivas visando à redução da morbimortalidade pela doença no país, através da melhoria dos indicadores de prevenção e diagnóstico por CCU.
\end{abstract}

Palavras-chave: Políticas de saúde, Neoplasia cervical, Atenção primária a saúde.

\begin{abstract}
Objective: To investigate public health policies aimed at the promotion and prevention of cervical cancer (CC), available in the literature from 2000 to 2020. Methods: This is an integrative review study of the National Library of Medicine (PUBMED), Scientific Electronic Library Online (SciELO) and Virtual Health Library (VHL) databases, published since 2000, in English, Portuguese and Spanish. Repeated works and those that did not answer the proposed research question were excluded. Then, four articles supported study. Results: It was possible to address the theme of cervical cancer, with regard to care management with an emphasis on primary health care, presenting the evolution of public policies implemented over the course of 20 years. Final considerations: The reviewed publications demonstrated that, over the period studied, there was a government effort to implement preventive measures aimed at reducing morbidity and mortality from the disease in the country, through the improvement of CC prevention and diagnosis indicators.
\end{abstract}

Key words: Health policy, Uterine cervical neoplasms, Primary health care.

\section{RESUMEN}

Objetivo: Investigar las políticas de salud pública orientadas a la promoción y prevención del cáncer cervicouterino (CCU), disponibles en la literatura de 2000 a 2020. Métodos: Se trata de un estudio de revisión integradora de las bases de datos de la National Library of Medicine (PUBMED), la Biblioteca Científica Electrónica en Línea (SciELO) y Bibioteca Virtual em Saúde (BVS), publicada desde 2000, en inglés, portugués y español. Se excluyeron los trabajos repetidos y aquellos que no respondieran a la pregunta de investigación propuesta. Resultados: Se logró abordar la temática del cáncer cervicouterino, en lo que respecta a la gestión de la atención con énfasis en la atención primaria de salud, presentando la evolución de las políticas públicas implementadas a lo largo de 20 años. Consideraciones finales: Las publicaciones revisadas demostraron que, durante el período estudiado, hubo un esfuerzo gubernamental para implementar medidas preventivas dirigidas a reducir la morbilidad y mortalidad por la enfermedad en el país, mediante la mejora de los indicadores de prevención y diagnóstico de CCU.

Palabras clave: Políticas de salud, Neoplasia cervical, Atención primaria de salud.

${ }^{1}$ Universidade Federal do Pará (UFPA), Belém - PA. *E-mail: lucrecia_cabral@hotmail.com

SUBMETIDO EM: 4/2021

ACEITO EM: 4/2021

PUBLICADO EM: 5/2021 


\section{INTRODUÇÃO}

Decorrentes das transições demográficas e epidemiológicas globais, as Doenças e Agravos não Transmissíveis (DCNT) já despontam como as principais causas de adoecimento e óbito da população mundial (WHO, 2018). Dentre as DCNT, o câncer apresenta um impacto cada vez maior nas taxas de incidência e mortalidade nas últimas décadas, por conta da dificuldade de acesso a medidas preventivas (FERLAY J, et al., 2018).

Em todo o mundo, a Organização Mundial de Saúde prevê que haverá um acréscimo $58 \%$ nos casos de câncer em 20 anos, passando de 18 milhões de casos em 2018, para 29 milhões em 2040. Na mesma medida, a mortalidade crescerá $67 \%$ no mesmo período, passando de 9 milhões para 16 milhões de mortes (GLOBOCAN, 2018).

No Brasil, a estimativa do Instituto Nacional de Câncer, para o ano de 2021, aponta a ocorrência de 625.370 casos novos de câncer, existindo grandes variações na distribuição por região geográfica. As regiões Sul e Sudeste têm maior prevalência de cânceres de próstata e de mama feminina, seguidos pelos cânceres de pulmão e de intestino. Ainda que similar, a região Centro-Oeste traz em seu perfil os cânceres do colo do útero e de estômago entre os mais incidentes. Ao passo que nas regiões Norte e Nordeste, tais cânceres denotam significativo impacto na população (INCA, 2020).

Entre os tumores malignos que mais acometem as mulheres, o câncer de colo de útero (CCU) figura como segundo câncer mais frequente no mundo, com 570.000 casos em 2018 (WHO, 2018). Anualmente, no Brasil são esperados 16.590 mil casos novos de CCU e um risco estimado de 15,43 a cada 100 mil mulheres. Na região Norte, esse risco aumenta para 21,20 a cada 100 mil mulheres, sendo a única região onde as taxas de incidência dos cânceres de mama e do colo do útero se equivalem entre as mulheres. Para o estado do Pará, em 2020, são estimados 780 casos para cada um destes (INCA, 2020).

A nível global, 311 mil mulheres morreram por CCU em 2018. As maiores taxas de mortalidade por CCU são encontradas na África oriental e central. Comparando-se os países das Américas, são mais elevadas em El Salvador, Nicarágua e Paraguai; e menores taxas encontram-se no Canadá, Porto Rico e Estados Unidos (DUARTE DAP e BUSTAMANTE-TEIXEIRA MT, 2018). As diferenças regionais ficam mais nítidas quando se comparam as taxas de incidência ajustadas segundo o Índice de Desenvolvimento Humano (IDH), em que o CCU ocupa o segundo lugar entre os mais incidentes e de maior mortalidade em países com baixo IDH (BRAY F, et al., 2018). É importante mencionar que o IDH retrata aspectos socioeconômicos de determinada região, através da análise da expectativa de vida ao nascer, da educação e do produto interno bruto. O CCU tem taxas de incidência inversamente proporcionais ao Produto Interno Bruto (SADOVSKY ADI, et al., 2015).

Em 28 países, a neoplasia mencionada no parágrafo anterior é a mais incidente, enquanto que em 42, é considerada a principal causa de morte, em que a maioria deles está localizada na África Subsaariana e no sudeste da Ásia. Em contraposição, o registro das taxas da referida neoplasia são de 7 a 10 vezes menores na América do Norte, Austrália, Nova Zelândia e leste da Ásia (BRAY F, et al., 2018). O Brasil apresenta taxas intermediárias, onde a mortalidade por CCU representou o terceiro lugar dos óbitos por câncer na população feminina em 2019. Contudo, no estado do Pará, há uma inversão das taxas, em que o CCU ocupa o primeiro lugar em óbitos (DATASUS, 2020).

O CCU possui lenta progressão, com fases bem delimitadas e um intervalo de 10 a 20 anos para se desenvolver, o que facilita a detecção precoce de alterações e, por conseguinte, propicia a prevenção e a cura da doença, por meio do diagnóstico rápido e do tratamento eficaz (BRITO-SILVA K, et al., 2014). Um meio bastante conhecido e eficiente de prevenção ao CCU é o exame citopatológico, também chamado de Papanicolau, que permite a identificação de lesões precursoras - passíveis de tratamento efetivo decorrentes de infecções persistentes pelo Papilomavírus Humano (HPV), considerado o principal fator de risco para o desenvolvimento da doença do câncer cervical (BARCELOS MRB, et al., 2017).

Outros importantes fatores de risco que impactam em maiores proporções de CCU são: coitarca precoce, múltiplos parceiros sexuais, multiparidade, tabagismo, déficits imunológicos, uso prolongado de contraceptivos orais, relações sexuais desprotegidas, higiene íntima precária (SOUZA AF e COSTA LHR, 
2015). O prognóstico do CCU varia conforme os seguintes fatores: extensão da doença e o tempo entre o diagnóstico e o início do tratamento. A mortalidade pela doença está diretamente relacionada ao seu diagnóstico em fase avançada e o início do tratamento tardio (CARVALHO PG, et al., 2018).

A escolha do método terapêutico depende do estadiamento da doença e de fatores pessoais, como idade e desejo da preservação da fertilidade. Os mais utilizados são cirurgia, radioterapia e quimioterapia, usados isoladamente ou em esquemas de tratamento que garantam maior eficácia e menor risco de recidiva (SADALLA JC, et al., 2015). Todas as modalidades devem ter abordagem multidisciplinar, a fim de permitir a efetividade no sucesso do manejo do paciente (BRASIL, 2013).

A partir desse prisma, a implantação de programas de rastreamento e controle do CCU em diversos países, como Canadá, Estados Unidos, Austrália, Finlândia, França, Suíça, Dinamarca, Irlanda e Holanda, demonstrou a redução em mais de 50\% na incidência da patologia (ARZUAGA-SALAZAR MA, et al., 2012).

Não obstante, as altas taxas de incidência, morbidade e mortalidade do CCU demonstram que a doença persiste como um problema de saúde pública no Brasil, a despeito de programas de detecção precoce, melhores tratamentos e maior conhecimento dos fatores de risco, que tem um valor intrínseco na análise das relações entre saúde, sociedade e ambiente. A partir desse contexto, o estudo objetivou investigar as políticas públicas de saúde voltadas para a promoção e prevenção do CCU no Brasil, disponíveis na literatura no período de 2000 a 2020.

\section{MÉTODOS}

Trata-se de Revisão Integrativa da Literatura (RIL) sobre as políticas públicas voltadas para o controle do câncer de colo uterino no Brasil. A RIL é caracterizada por agregar, sintetizar e discutir informações essenciais de cunho científico para fomentar determinadas temáticas. O levantamento dos dados ocorreu no mês de janeiro de 2021.

O levantamento da produção científica baseou-se nas seguintes etapas: escolha da problemática e pergunta de pesquisa; seleção de critérios para obtenção da amostra; inclusão de artigos relevantes ao tema; análise de artigos; discussão e resultados sintetizados e apresentação da revisão integrativa (MENDES KDS, et al., 2008).

A etapa de formulação da pergunta de pesquisa baseou-se na estratégia PICO, onde P(Problema) I(interesse), Co(Contexto), sendo o P: CCU, I: políticas públicas, Co: atenção primária em saúde, suscitando a pergunta norteadora: qual a produção bibliográfica sobre as políticas públicas de saúde direcionadas à promoção e prevenção do CCU no Brasil? A partir disso, selecionaram-se os Descritores em Ciências da Saúde (DECS): Health Policy, Uterine Cervical Neoplasms, Primary Health Care, em associação com o operador booleano $A N D$.

A procura por artigos científicos ocorreu nas bases/bancos de dados eletrônicas: Biblioteca Virtual em Saúde (BVS), National Library of Medicine (PUBMED) e Scientific Electronic Library Online (SciELO). Para a obtenção da amostra, utilizaram-se como critérios de inclusão: artigos completos e disponíveis na integra; publicados no período de janeiro/2000 a dezembro/2020; nos idiomas inglês, português e espanhol.

Aplicaram-se como critérios de exclusão trabalhos duplicados, resumos de eventos, anais, dissertações, teses, artigos que não se enquadravam no recorte temporal ou que não respondiam à questão de pesquisa deste estudo.

Inicialmente, foram encontrados 190 artigos. Após a leitura dos títulos e resumos, foram excluídos por 60 duplicidade; 105 retirados por não responder à pergunta de pesquisa. Desta forma, 25 artigos foram selecionados para a leitura na íntegra e verificar a possibilidade de inclusão na pesquisa. Por conseguinte, 0 estudo foi subsidiado por quatro artigos. A ilustração completa do fluxograma adotado no estudo está descrita na Figura 1. 
Figura 1 - Fluxograma sobre procedimento metodológico de seleção dos estudos.

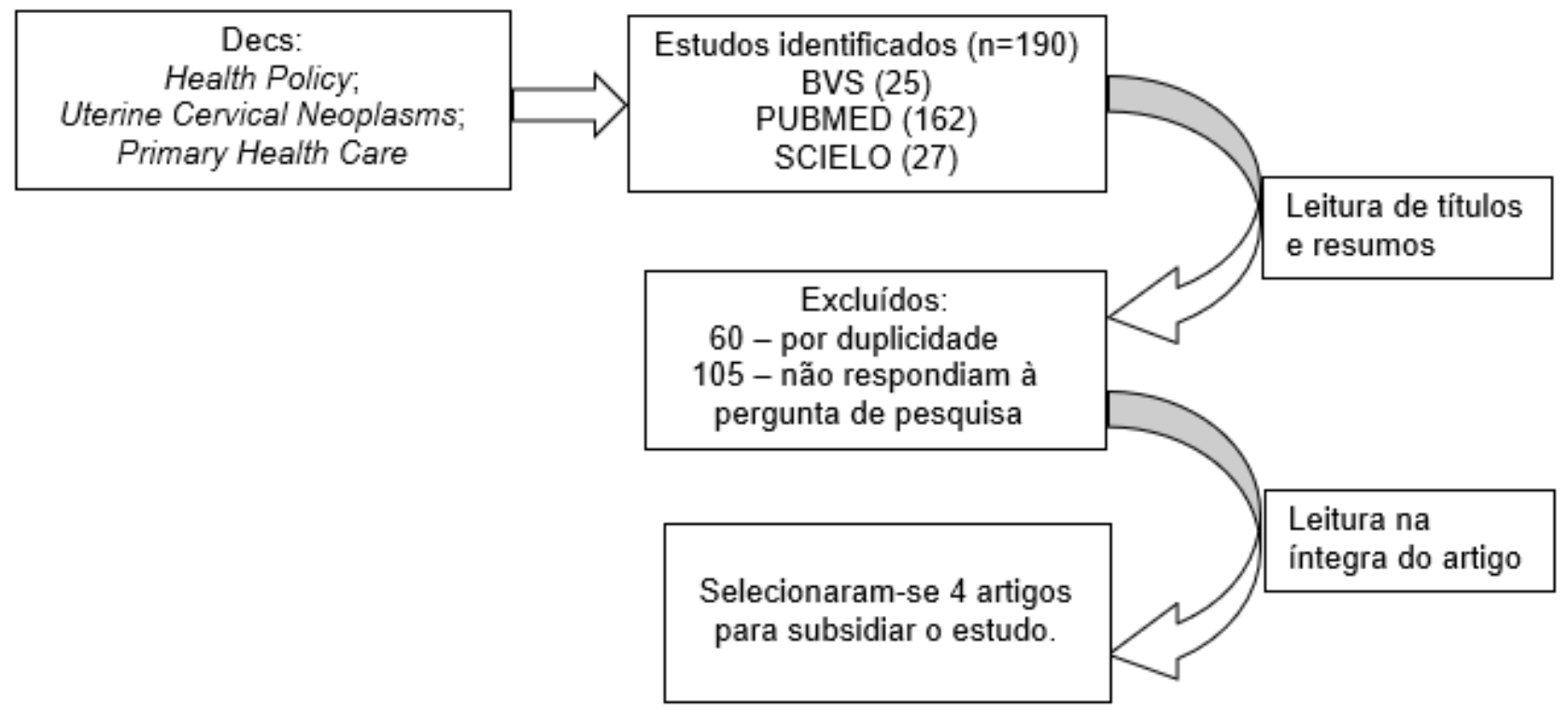

Fonte: Formigosa LAC e Silva MVS, 2021.

A coleta da amostra guiou-se pelo formulário validado por Ursi, adaptado pelos autores, contendo as variáveis: Autor, ano e principais resultados, sendo que os dados obtidos foram analisados de forma descritiva, originando um quadro sinóptico de caracterização dos estudos (URSI ES, 2005).

\section{RESULTADOS E DISCUSSÃO}

Ao final da análise crítica das publicações, foram encontrados apenas três artigos que revisitaram as políticas públicas no Brasil, no que concerne às ações voltadas ao câncer de colo de útero (Quadro 1).

Quadro 1 - Síntese dos resultados dos artigos selecionados na revisão.

\begin{tabular}{|c|c|l|}
\hline N & Autores (ano) & \multicolumn{1}{c|}{ Principais Resultados } \\
\hline 1 & TSUCHIYA CT, et al. (2017) & $\begin{array}{l}\text { Estudo de revisão. No Brasil, políticas públicas voltadas para o CCU } \\
\text { vêm sendo desenvolvidas desde a década de 1970 e incluíram } \\
\text { diversos programas de rastreamento, que ampliaram a cobertura do } \\
\text { exame preventivo, entretanto, a doença permanece como problema } \\
\text { de saúde pública. }\end{array}$ \\
\hline 2 & SILVA MJS, et al. (2017) & $\begin{array}{l}\text { Estudo descritivo. As sucessivas normativas permitiram a } \\
\text { estruturação de uma política de atenção ao câncer com vários } \\
\text { elementos, consoante a relevância epidemiológica da doença e o } \\
\text { destaque dado durante o período. No entanto, o grande volume de } \\
\text { normas dificulta o conhecimento dos gestores e profissionais de } \\
\text { saúde, ainda que inseridos e atuantes na área. }\end{array}$ \\
\hline 3 & TEIXEIRA LA, et al. (2012) & $\begin{array}{l}\text { Estudo histórico social. A partir da análise documental, traz uma } \\
\text { abordagem histórica da saúde pública no Brasil voltada ao câncer e } \\
\text { as transformações ocorridas entre os anos de 1900 e 2011, } \\
\text { passando do entendimento de problema de saúde individual para } \\
\text { integrar a agenda institucional brasileira. }\end{array}$ \\
\hline 4 & PARADA R, et al. (2008) & $\begin{array}{l}\text { Estudo descritivo. Apresenta um panorama do controle do câncer no } \\
\text { Brasil, com destaque para a atenção primária na detecção precoce } \\
\text { dos cânceres de mama e do colo do útero, apontando a necessidade } \\
\text { de qualificação da rede assistencial para a integralidade e } \\
\text { efetividade as estratégias das ações implantadas. }\end{array}$ \\
\hline
\end{tabular}

Fonte: Formigosa LAC e Silva MVS, 2021. 
Durante a primeira metade do XX, as iniciativas com vistas ao controle do câncer no Brasil foram desenvolvidas de maneira isolada por instituições filantrópicas e faculdades de medicina, fora da esfera pública. Entretanto, o cenário epidemiológico do CCU no país, em conjunto com o processo de redemocratização política do país e o anseio da população pela maior presença do Estado nas políticas de saúde, demonstraram a magnitude da doença e reafirmou a necessidade de implantação de ações amplas e abrangentes em âmbito nacional (TEIXEIRA LA, et al., 2012; TSUCHIYA CT, et al., 2017).

As discussões sobre tal necessidade foram impulsionadas na década de 70 com o debate trazido à tona pela Organização Pan-Americana de Saúde (OPAS), que resultou na criação do Programa Nacional de Controle do Câncer; e pelo movimento das mulheres, que incorporou Programa de Atenção Integral à Saúde da Mulher (INCA, 2018).

A partir da Constituição Federal de 1988 e da consequente criação do Sistema Único de Saúde (SUS), o Instituto Nacional do Câncer (INCA) passou a coordenar a formulação das políticas públicas com ênfase no cuidado integral, respeitando os princípios de universalidade, integralidade; além de contemplar ações nas esferas de informação, educação e prevenção (TEIXEIRA LA, et al., 2012).

Destarte, a consolidação do SUS tornou possível a instituição de mecanismos que permitiram a organização da atenção oncológica no Brasil de modo que a partir do final dos anos 1990, uma série de medidas normativo-legais foi aplicada com o intuito de atingir esses objetivos (PARADA R, et al., 2008). Em 1995, após a IV Conferência Mundial sobre a Saúde da Mulher, foi elaborado o projeto piloto "Viva Mulher", implantado em quatro cidades no país (Belém, Curitiba, Recife, Rio de Janeiro), além do Distrito Federal, e tinha como intuito a padronização da coleta de material para o seguimento e conduta frente a cada alteração citológica (INCA, 2016).

Com base nos resultados desse projeto piloto, foi instituído o Programa Nacional de Combate ao Câncer de Colo do Útero em 1998, que objetivou ampliar o número de exames citopatológicos realizados no país na faixa etária definida como de risco (INCA, 2018). Essa fase teve como marco legal a Portaria MS/GM no 3.535/1998, que definiu um parâmetro epidemiológico/ demográfico (casos novos anuais de câncer) para justificar a necessidade de novos serviços de alta complexidade em oncologia, estabelecendo uma rede hierarquizada. Concomitantemente, tornou obrigatório o funcionamento dos Registros Hospitalares de Câncer nestes serviços, para oportunizar a avaliação da assistência prestada ao paciente oncológico (SILVA MJS, et al., 2017).

Com o propósito de que houvesse o monitoramento e gerenciamento das ações desenvolvidas pelo Programa mencionado anteriormente, em relação ao rastreamento da doença, foi criado em 1999 o Sistema de Informação do Câncer de Colo de Útero (SISCOLO) (INCA, 2016).

Ainda nesse período, houve a criação de um incentivo financeiro visando à ampliação do número de registros de câncer brasileiros, através do Programa de Avaliação e Vigilância do câncer e seus fatores de risco, o que propiciou um aumento de $100 \%$ do número de Registros de Câncer de Base Populacional no país, consolidando-os como pilares fundamentais à vigilância do câncer para planejamento e avaliação das ações de controle do câncer, a partir do monitoramento do perfil das neoplasias em determinada área geográfica (BRASIL, 2007).

Em 2003, objetivando uniformizar a terminologia utilizada em laudos citopatológicos no Brasil, assim como aprimorar a qualidade destes exames, foi publicada a Nomenclatura Brasileira para Laudos Citopatológicos Cervicais, atualizada pela última vez em 2012. A publicação trouxe como contribuições a incorporação de conceitos clínicos, morfológicos e moleculares; e a utilização do sistema Bethesda adaptado, o que permite a comparabilidade de resultados nacionais aos internacionais (INCA, 2012).

Em substituição à política anterior, foi estabelecida a Política Nacional de Atenção Oncológica (PNAO) Portaria GM/MS n²439/2005, que de forma inovadora, possibilitou a organização da linha de cuidados do paciente com câncer, integrando as ações nas três esferas de governo e em todos os níveis de atenção à saúde: promoção da saúde, detecção precoce, tratamento, vigilância em saúde, formação de recursos humanos, comunicação e mobilização social, pesquisa e gestão do SUS (BRASIL, 2005). 
No contexto da PNAO, a Rede de Atenção Oncológica (RAO), regulamentada pela Portaria $n^{\circ} 741 / 2005$, mudou o processo de habilitação dos serviços oncológicos, passando a defini-los como Centros e Unidades de Assistência de Alta Complexidade em Oncologia (CACON e UNACON).

A partir de 2006, com a Portaria 399/06, que instituiu o Pacto pela Saúde, as ações de prevenção, detecção precoce e tratamento oportuno passam a constar nos planos de saúde dos estados e municípios, de modo que cada esfera governamental passa ter gestão sobre a RAO de sua responsabilidade (CARVALHO BG, et al., 2015).

A transversalidade das ações, nesse período, pode ser percebida pela inclusão do controle do CCU como prioridade em outras políticas de saúde: Pacto pela Vida, Política Nacional de Atenção Básica, Política de Atenção Integral à Saúde da Mulher e Política Nacional de DST/Aids (PARADA R, et al., 2008).

O CCU também aparece como prioridade no Plano de Ações Estratégicas para o Enfrentamento das Doenças Crônicas não Transmissíveis (DCNT) no Brasil, 2011-2022, o qual aborda o cuidado integral e prevê a ampliação para $85 \%$ da cobertura de exame citopatológico em mulheres de 25 a 64 anos; além de tratamento de $100 \%$ das mulheres com diagnóstico de lesões precursoras de câncer (BARCELOS MRB, et al., 2017).

Em 2011, foram lançadas as Diretrizes Brasileiras para Rastreamento do CCU, com atualização em 2016, resultado de trabalho conjunto de várias instituições governamentais e de cunho científico. Tais Diretrizes têm o intuito de auxiliar os profissionais da saúde nas práticas assistenciais e apoiar os gestores na tomada de decisão em relação à organização e à estruturação da linha de cuidados da mulher com CCU (INCA, 2016).

Nesse sentido, a principal estratégia de rastreamento adotada pelo Brasil foi a realização do exame citopatológico, o qual deve estar disponível e de fácil acesso na Atenção Primária à Saúde (APS), fazendo parte da organização dos serviços (PARADA R, et al., 2008). O exame deve ser realizado anualmente e após dois resultados negativos consecutivos, a cada 3 anos. É indicado para mulheres a partir de 25 anos, com vida sexual ativa, até os 64 anos de idade (INCA, 2016).

Outro importante marco no enfrentamento da doença foi a promulgação da Lei $n^{\circ} 12.732 / 2012$, a qual prevê o prazo de 60 dias, a contar do diagnóstico, para iniciar o tratamento pelo Sistema Único de Saúde, tempo considerado aceitável para efeitos de progressão, classificação e prognóstico da doença (BRASIL, 2012). Recentemente, alterada pela Lei $n^{\circ}$ 13.896/2019, que determina a realização do diagnóstico de caso suspeito de neoplasia maligna em até 30 dias (BRASIL, 2019).

Somente em 2013, a PNAO foi substituída pela Política Nacional para a Prevenção e Controle do Câncer (PNPCC), através da Portaria GM/MS no 874 de 2013, que manteve as diretrizes relacionadas às linhas de cuidado, e deu maior ênfase à integralidade do cuidado e à informação em saúde, resultando na estruturação de serviços voltados ao diagnóstico e tratamento de lesões precursoras do CCU - SRC (Portaria no 189/14), assim como na implantação do Sistema de Informação do Câncer - SISCAN (Portaria no 3394/13) (SILVA MJS, et al., 2017).

Para organizar o sistema, novos critérios e parâmetros passaram a ser estabelecidos pela Portaria ํo 140/2014. Tal redefinição buscou ampliar a qualificação e consolidar a atuação dos serviços especializados em oncologia. O parâmetro para habilitação de UNACON e CACON passou a adotar o número de habitantes de cada região (uma unidade para 500 mil).

No mesmo ano, houve a redefinição da Qualicito - Qualificação Nacional em Citopatologia, na prevenção do CCU no âmbito da Rede de Atenção à Saúde das Pessoas com Doenças Crônicas, por meio da Portaria GM/MS no 1504 de 23 de julho de 2013, que permite a avaliação de desempenho dos laboratórios públicos e privados prestadores de serviço ao SUS (INCA, 2016).

Outra vertente implantada com vistas à prevenção do CCU, foi a inclusão no ano de 2014 da vacina quadrivalente contra o Papilomavirus Humano (HPV), no Programa Nacional de Imunização, oferecendo proteção contra os tipos $6,11,16$ e 18, inicialmente com público alvo de meninas na faixa etária de 9 a 14 anos, ampliado em 2017 para meninos entre 11 e 14 anos e grupos com condições clínicas especiais (homens 
e mulheres de 9 a 26 anos vivendo com HIV/AIDS; indivíduos transplantados por órgãos sólidos e medula óssea; e pacientes oncológicos) (BRASIL, 2018).

Nesse aspecto, faz-se mister ressaltar que a infecção persistente pelo HPV é considerada como causa necessária, mas não suficiente para o surgimento da neoplasia. Daí o programa de vacinação contra o HPV ser extremamente significativo na redução a longo prazo das taxas de $\mathrm{CCU}$, pois tem efeito profilático diminuindo entre 70 a $90 \%$ de todos os cânceres cervicais, sendo inclusive recomendado pela Organização Mundial de Saúde, por representar uma das intervenções com melhor custo-efetividade (BRAY F, et al., 2018).

Em 2019, houve a redefinição dos critérios e parâmetros referenciais para a habilitação de estabelecimentos de saúde na alta complexidade em oncologia no âmbito do SUS, Portaria SAES/MS no 1399/2019. O objetivo da portaria foi buscar uma maior uniformização dos parâmetros e adequar as inconsistências da Portaria anterior SAS/MS no 140/2014 (BRASIL, 2019).

Em relação ao estado do Pará, temos como marco a aprovação do Plano Estadual e da RAO pelo Conselho Estadual de Saúde e Comissão Intergestores Bipartite, por meio das Resoluções n 034/2015 e 108/2015. Outro relevante acontecimento foi a criação da Câmara Técnica de Oncologia e do Comitê de Apoio e Controle Social ao Câncer no estado do Pará, por meio das Portarias no 884/2015 e 316/2017, respectivamente, as quais possibilitaram a discussão dos problemas relacionados à oncologia com todos os envolvidos da área técnica e da sociedade civil organizada (SESPA, 2018).

Por todo o exposto, fica claro o esforço governamental no sentido de nortear o controle do CCU por meio da linha de cuidados, com a definição de fluxo assistencial e da rede de serviços, e a organização das ações nos três níveis hierárquicos do SUS, a fim de garantir a integralidade da atenção à saúde (LOPES VAS e RIBEIRO JM, 2019).

Nesta perspectiva, as linhas de cuidado são estratégias de estabelecimento da trajetória assistencial, com o objetivo de organizar o fluxo de usuários conforme as necessidades. Implicam na organização de um conjunto de ações e serviços de saúde, estruturados a partir de critérios epidemiológicos e de regionalização para atender aos desafios que apresentem relevância epidemiológica e social (BRASIL, 2013; TSUCHIYA CT, et al., 2017).

Especialmente em relação ao CCU, a linha de cuidados tem a finalidade de assegurar à mulher o acesso humanizado e integral às ações e aos serviços, a fim de propiciar prevenção da doença e acesso ao rastreamento das lesões precursoras, ao diagnóstico precoce e ao tratamento adequado, qualificado e em tempo oportuno (CARVALHO PG, et al., 2018).

Tais ações impactam diretamente na APS, por ser responsável pelo acompanhamento longitudinal das usuárias, mesmo quando estão em outro nível de assistência. Ademais, deve coordenar e articular os serviços e unidades de saúde que compõem a RAO, participando na definição dos fluxos e contrafluxos e na determinação das prioridades da população (BRASIL, 2013).

\section{CONSIDERAÇÕES FINAIS}

A análise dos dados possibilitou verificar que nas últimas décadas várias ações foram implantadas com vistas a organizar os serviços oncológicos, orientando a formação de redes regionais, com fluxo de acesso regulado e garantindo serviços desde a atenção primária à alta complexidade. Nesse sentido, é de extrema importância o desenvolvimento de mais estudos que demonstrem a mudança na concepção do processo saúde-doença e o reflexo disto no planejamento da linha de cuidados e da rede de atenção oncológica.

\section{REFERÊNCIAS}

1. ARZUAGA-SALAZAR MA, et al. El cáncer de cuello de útero: un problema social mundial. Revista Cubana de Enfermería, 2012; 28(1);63-73.

2. BARCELOS MRB, et al. Quality of cervical cancer screening in Brazil: external assessment of the PMAQ. Revista de Saúde Pública, 2017; 51(67):1-13. 
3. BRASIL. 2005. In: PORTARIA N²439/GM, DE 08 DE DEZEMBRO DE 2005: Institui a Política Nacional de Atenção Oncológica: Promoção, Prevenção, Diagnóstico, Tratamento, Reabilitação e Cuidados Paliativos, a ser implantada em todas as unidades federadas, respeitadas as competências das três esferas de gestão.

4. BRASIL. Diário Oficial da União. Lei no 12.732, de 22 de novembro de 2012. Dispõe sobre o primeiro tratamento de paciente com neoplasia maligna comprovada e estabelece prazo para seu início. Brasil: Ministério da Saúde, 2012.

5. BRASIL. Diário Oficial da União. Lei no 13.896 , de 30 de outubro de 2019. Altera a Lei no 12.732 , de 22 de novembro de 2012, para que os exames relacionados ao diagnóstico de neoplasia maligna sejam realizados no prazo de 30 (trinta) dias, no caso em que especifica. Brasil: Ministério da Sáude, 2019.

6. BRASIL. Ministério da Saúde. Controle dos cânceres do colo do útero e da mama. Secretaria de Atenção à Saúde. Departamento de Atenção Básica. - 2. ed. - Brasília: Ministério da Saúde, 2013.

7. BRASIL. Ministério da Saúde. Departamento de Ciência e Tecnologia e Insumos Estratégicos. Integração de informações dos registros de câncer brasileiros. Rev. Saúde Pública, 2007; 41(5):865-868

8. BRASIL. Ministério da Saúde. Informe técnico da ampliação da oferta das vacinas papilomavírus humano 6,11, 16 e 18 (recombinado) - vacina HPV quadrivalente e meningocócica C (conjugada). Brasília: março, 2018. Disponível em: <portalarquivos 2.saude.gov.br/images/pdf/2018/marco/14/Informe-T—cinco-HPV-MENINGITE.pdf> Acesso em: 25 jan. 2020.

9. BRASIL. 2019. PORTARIA SAES/MS № 1399, DE 17 DE DEZEMBRO DE 2019: Redefine os critérios e parâmetros referenciais para a habilitação de estabelecimentos de saúde na alta complexidade em oncologia no âmbito do SUS.

10. BRAY F, et al. Global Cancer Statistics 2018: GLOBOCAN Estimates of Incidence and Mortality Worldwide for 36 Cancers in 185 Countries. Cancer Journal for Clinicians, 2018; 68(6):394-424.

11. BRITO-SILVA K, et al. Integralidade no cuidado ao câncer do colo do útero: avaliação do acesso. Rev Saúde Pública. 2014; 48(2):240-248.

12. CARVALHO BG, et al. Integralidade do cuidado no Programa de Controle do Câncer de Colo Uterino: visão das usuárias com alteração na citologia oncótica. Saúde em Debate, 2015; 39(106):707-717.

13. CARVALHO PG, et al. Trajetórias assistenciais de mulheres entre diagnóstico e início de tratamento do câncer de colo uterino. Saúde em Debate, 2018; 42(118):687-701.

14. BRASIL. Ministério da Saúde. Departamento de informática do Sistema Único de Saúde. Sistema de Informação sobre Mortalidade. Disponível em: tabnet.datasus.gov.br. Acesso em: 12 jan. 2021.

15. DUARTE DAP, BUSTAMANTE-TEIXEIRA MT. Iniquidade Social e Mortalidade por Câncer de Mama e Colo do Útero: Uma Revisão Integrativa. Rev Fund Care Online, 2018; 10(3):877-888.

16. FERLAY J, et al. (ed.). Cancer today. Lyon, France: International Agency for Research on Cancer, 2018. (IARC CAncerBase, n. 15). Disponível em: https://publications.iarc.fr/Databases/larc-Cancerbases/Cancer-Today-PoweredBy-GLOBOCAN-2018--2018. Acesso em: 10 dez. 2020.

17. GLOBOCAN, 2018. Cancer Tomorrow. Cancer Incidence and Mortality Worldwide: IARC Cancer Base no. 112018. Disponível em http://globocan.iarc.fr/Default.aspx. Acesso em: 20 jan 2021.

18. INSTITUTO NACIONAL DE CÂNCER. Coordenação-Geral de Prevenção e Vigilância. Divisão de Detecção Precoce e Apoio à Organização de Rede. Nomenclatura brasileira para laudos citopatológicos cervicais. 3th. ed. Rio de Janeiro: Inca, 2012. 23p.

19. INSTITUTO NACIONAL DE CÂNCER. Coordenação de Prevenção e Vigilância. Divisão de Detecção Precoce e Apoio à Organização de Rede. Diretrizes brasileiras para o rastreamento do câncer do colo do útero. 2 nd. ed. rev. atual. Rio de Janeiro: INCA, 2016.

20. INSTITUTO NACIONAL DE CÂNCER. Viva Mulher 20 anos: história e memória do controle do câncer do colo do útero e de mama no Brasil: catálogo de documentos. Rio de Janeiro: INCA, 2018.

21. INSTITUTO NACIONAL DE CÂNCER. Coordenação de Prevenção e Vigilância. Estimativa 2020: Incidência do Câncer no Brasil. 2020.

22. LOPES VAS, RIBEIRO JM. Fatores limitadores e facilitadores para o controle do câncer de colo de útero: uma revisão de literatura. Ciência e Saúde Coletiva, 2019; 24(9):3431-3442.

23. MENDES KDS, et al. Revisão integrativa: método de pesquisa para a incorporação de evidências na saúde e na enfermagem. Texto contexto, 2008; 17(4):758-764.

24. PARADA R, et al. A Política Nacional de Atenção Oncológica e o papel da Atenção Básica na prevenção primária e controle do câncer. APS, 2008; 11(2):199-206.

25. SADALLA JC, et al. Cervical cancer: what's new? Rev Assoc Med Bras, 2015; 61(6):536-542.

26. SADOVSKY ADI, et al. Índice de Desenvolvimento Humano e prevenção secundária de câncer de mama e colo de útero: um estudo ecológico. Cad Saúde Pública, 2015; 31(7):1539-1550.

27. SESPA. Secretaria de Estado de Saúde Pública do Pará. Diretoria de Desenvolvimento das Redes Assistenciais. Coordenação Estadual de Atenção Oncológica. Plano Estadual de Atenção Oncológica do Estado do Pará. 20152018. Belém: SESPA, 2018.

28. SOUZA AF, COSTA LHR. Conhecimento de Mulheres sobre HPV e Câncer do Colo do Útero após Consulta de Enfermagem. Revista Brasileira de Cancerologia, 2015; 61(4):343-50.

29. SILVA MJS, et al. Política de Atenção ao Câncer no Brasil após a Criação do Sistema Único de Saúde. Revista Brasileira de Cancerologia, 2017; 63(3):177-187.

30. TEIXEIRA LA, et al. Políticas públicas de controle de câncer no Brasil: elementos de uma trajetória Cad. Saúde Colet., 2012; 20(3):375-380.

31. TSUCHIYA CT, et al. O câncer de colo do útero no Brasil: uma retrospectiva sobre as políticas públicas voltadas à saúde da mulher. J Bras Econ Saúde, 2017; 9(1):137-147.

32. URSI ES. Prevenção de lesões de pele no perioperatório: revisão integrativa da literatura. Dissertação (Mestrado em Enfermagem) - Escola de Enfermagem, Universidade de São Paulo, Ribeirão Preto, 2005. 130p.

33. WORLD HEALTH ORGANIZATION (WHO). Global Health Observatory. Genebra: World Health Organization, 2018. Disponível em http://www.who.int/healthinfo/global_burden_dis ease/en/. Acesso em: 15 dez. 2020. 